\title{
Evaluation of magnetic resonance imaging issues of titanium and stainless steel brackets
}

\author{
Igor Linetskiy a,*, Jana Starčukováb, Hana Hubálkovác, Zenon Starčuk Jr ${ }^{\mathrm{b}}$, Mutlu Özcan ${ }^{\mathrm{d}}$ \\ a Department of Oral and Maxillofacial Surgery, First Faculty of Medicine, Charles University, Prague, \\ Czech Republic \\ b Department of Magnetic Resonance and Cryogenics, Institute of Scientific Instruments, \\ Czech Academy of Sciences, 61264 Brno, Czech Republic \\ c Department of Prosthodontics, Department of Stomatology, First Faculty of Medicine, Charles University, \\ Prague, Czech Republic \\ d Dental Materials Unit, Centre for Dental and Oral Medicine, \\ Clinic for Fixed and Removable Prosthodontics and Dental Materials Science, University of Zürich, \\ Zürich, Switzerland
}

*Corresponding author, e-mail: igor.linetskiy@gmail.com

Received 20 Feb 2018

Accepted 8 Apr 2019

\begin{abstract}
Magnetic resonance imaging (MRI) is a sophisticated diagnostic method of contemporary medicine. This examination in patients with metallic objects is often complicated due to MRI issues. Clinically significant difference in MRI issues of titanium (equilibrium ti, Dentaurum) and stainless steel (Gemini, 3M Unitec) brackets was studied in a clinical 1.5 T and an experimental 4.7 T MRI scanners. In this in vitro study, the following parameters were assessed: artefacts, magnetic field interaction, and heating. Artefacts were evaluated in spin and gradient echo images using ASTM F2119-07 standard in 1.5 T field. Translational attraction, torque and heating were tested. The titanium bracket for upper lateral incisor produced $2.5-4.3 \mathrm{~mm}$ and $4.7-4.9 \mathrm{~mm}$ artefacts in spin and gradient echo scans, respectively. The stainless steel bracket for upper lateral incisor caused more than $50 \mathrm{~mm}$ distortions in the same sequences. Images of the titanium bracket set were diagnostically acceptable whereas the stainless steel bracket set could not be graded due to the incapability of the MRI scanner to adjust resonance frequency. No clinically relevant translational attraction and no torque were detected for equilibrium ti brackets, while Gemini brackets were strongly attracted by the magnetic field. No clinically significant heating was observed. Noticeable difference in MRI issues of the brackets was observed in artefacts and mobility. Gemini brackets were found unacceptable for MRI.
\end{abstract}

KEYWORDS: MRI issues, artefact, magnetic field interaction, heating, orthodontic bracket

\section{INTRODUCTION}

Magnetic resonance imaging (MRI) is of significant importance for medical diagnostics. Since its first application in 1980's, over 200 million MRI examinations have been performed in patients of all age groups. Its advantages comprise safety (absence of ionizing radiation), non-invasiveness, good differentiation of hard and especially soft tissues ${ }^{1-4}$. Tumours of the head and neck area, TMJ conditions, seizure disorders, aneurysms, Alzheimer's disease and migraine are generally diagnosed with the aid of this method ${ }^{2,5,6}$.

The latest trend in dentistry is absolute aesthetics. It is usually reached by orthodontic treatment realized with a fixed appliance, which is mostly composed of brackets, bands, arches, wires, and other components. Originally, brackets were manufactured from a variety of stainless steel (SS) alloys (UNS S30300, S30400, S31600, S17400, etc). Recently, new materials, such as titanium and its alloys, cobalt-chromium alloys, and gold alloys, were introduced. Public demand for high aesthetics caused ceramic and plastic brackets to become widely spread ${ }^{7}$.

Along with the advantages, MRI has certain shortcomings. One of them is the interaction between the magnetic resonance (MR) scanner magnetic field and metal materials ${ }^{8}$. This interference can manifest itself as follows: artefacts $^{9-14}$; mechanic effects (translational attraction and torque $)^{9,15-17}$; heating ${ }^{15-18}$; device or item activation, deactivation, or damage caused by the MR system's magnetic or radiofrequency (RF) fields; 
false information presented on an electrically active device caused by the MR system's static, switching or RF magnetic fields; patient nerve stimulation ${ }^{18}$.

Interference of medical devices with MRI was until recently defined by FDA in Ref. 19 using two subcategories - MR Safe and MR Compatible. Contemporary ASTM F2503-08 standard classifies them as MR Safe, MR Unsafe, and MR Conditional ${ }^{15}$. Artefacts on MR images are no longer considered by this standard.

In orthodontic patients, radiologists mostly refuse to perform MRI because of fear of adverse interactions of metal devices with the strong magnetic field of the MRI scanner. All orthodontic metal components are preferred to be removed prior to examination, which is harmful to hard dental tissues, time-consuming and expensive. Another possibility is to choose alternative diagnostic method but MRI is irreplaceable in the majority of cases. Various studies of dental alloys indicate that there is an enormous difference in their MR issues because the material composition differs significantly ${ }^{13,20-22}$. Majority of studies on orthodontic appliances showed that SS brackets caused moderate to significant MR image distortions ${ }^{23-28}$. MR safety studies reported negligible heating and no risk of displacement for properly fixed brackets ${ }^{29-32}$. Since most orthodontists prefer SS brackets $(86 \%$ and $95 \%$ of the specialists in the US and the UK, respectively) ${ }^{33,34}$, patients with these brackets are not acceptable for MRI.

Magnetic induction of the static magnetic field is one of the factors determining the clinical impact of MRI issues. The fields of MR scanners range from 0.2-9.4 T, with over $200001.5 \mathrm{~T}$ systems in use nowadays ${ }^{35,36}$. Furthermore high-field MRI at $3 \mathrm{~T}$ or higher has become more common in daily practice.

Quantification of the artefacts and their distortive effect on the images is not easy to describe. Magnetic susceptibility of the material producing the artefact is the major indicator of the potential adverse effects ${ }^{13,20}$. Large image distortions were reported in the presence of base metal dental alloys whereas precious metal and titanium alloys have been found to be acceptable in $\mathrm{MRI}^{20,32}$. As far as we know, there has been no standard measurement of the artefacts caused by ferromagnetic and non-ferromagnetic brackets and their quantitative comparison. Furthermore, validation of MR safety of two basic types of bracket materials is of great importance.

The aim of this study was to measure the arte-
Table 1 Elemental compositions, size and manufacturer information of the materials.

\begin{tabular}{|c|c|c|c|}
\hline Brand name & Size (mm) & Composition & Manufacturer \\
\hline Gemini & $\begin{array}{l}\text { In/Out: } 1.3 \\
\text { Width: } 3.0\end{array}$ & $\mathrm{~N} / \mathrm{A}$ & 3M Unitec ${ }^{\mathrm{a}}$ \\
\hline Equilibrium ti & $\begin{array}{l}\text { various } \\
\text { shapes }\end{array}$ & $\begin{array}{l}\text { Commercially } \\
\text { pure titanium }\end{array}$ & Dentaurum $^{\mathrm{b}}$ \\
\hline
\end{tabular}

a 3M Unitec, Monrovia, California, USA.

${ }^{\mathrm{b}}$ Dentaurum, Ispringen, California, USA.

Table 2 Pulse sequence parameters.

\begin{tabular}{|c|c|c|c|}
\hline \multirow[t]{2}{*}{ Slice } & \multicolumn{2}{|c|}{ Single bracket } & \multirow{2}{*}{$\frac{\text { Brackets }}{\mathrm{SE}^{*}}$} \\
\hline & $\mathrm{GE}^{*}$ & SE & \\
\hline Bandwidth & $32 \mathrm{kHz}$ & $32 \mathrm{kHz}$ & $32 \mathrm{kHz}$ \\
\hline $\begin{array}{l}\text { Field of view } \\
(\mathrm{mm} \times \mathrm{mm})\end{array}$ & $120 \times 120$ & $120 \times 120$ & $\begin{array}{l}160 \times 160^{\mathrm{S}, \mathrm{C}} \\
120 \times 120^{\mathrm{T}}\end{array}$ \\
\hline Matrix size & $256 \times 256$ & $256 \times 256$ & $256 \times 256$ \\
\hline Thickness & $3 \mathrm{~mm}$ & $3 \mathrm{~mm}$ & $3 \mathrm{~mm}$ \\
\hline $\mathrm{TR}$ (ms) & 200 & 3000 & 3000 \\
\hline $\mathrm{TE}(\mathrm{ms})$ & $\begin{array}{c}10 \\
6^{S}\end{array}$ & 15 & 15 \\
\hline No. slices & 21 & 21 & 21 \\
\hline $\begin{array}{l}\text { Gradient } \\
\text { (Hz/pixel) }\end{array}$ & $\begin{array}{l}120 \\
310^{S}\end{array}$ & 125 & 125 \\
\hline Orientation $^{\dagger}$ & S,T,C & S,T,C & S,T,C \\
\hline
\end{tabular}

' S, sagittal; C, coronal; T, transversal.

* S,T,C superscripts indicate for the particular orientations of slices.

facts of two selected bracket sets: equilibrium ti (Dentaurum) and Gemini (3M Unitec) fabricated of different metal alloys, using the modified ASTM F2119-07 standard ${ }^{37}$, and to estimate their magnetic field interaction and heating to demonstrate that selection of more acceptable material can significantly improve the diagnostic accuracy of orofacial, TMJ and brain imaging, and provide MR safety.

\section{METHODS}

Maxillary equilibrium ti (commercially pure titanium) and Gemini (stainless steel) orthodontic brackets were employed in this in vitro study. Elemental compositions, size and manufacturers information are summarized in Table 1. The whole sets mounted on a jaw model were tested for MR image artefacts. A single bracket for maxillary right lateral incisor from each set was tested for both MR 
Table 3 Modified receiver operating characteristic method of distortion classification.

\begin{tabular}{ll}
\hline Image appearance & Diagnostic \\
\hline No distortion/artefact & Diagnostic \\
Minimal distortion/artefact & Diagnostic \\
Moderate distortion/artefact & Moderately diagnostic \\
Severe distortion/artefact & Nondiagnostic \\
Complete obliteration/artefact & Nondiagnostic \\
\hline
\end{tabular}

artefacts and MR safety.

The imaging was performed in a $1.5 \mathrm{~T}$ wholebody scanner (MAGNETOM Symphony, Siemens AG). Multislice turbo spin echo (SE) and gradient echo (GE) images covering the extent of the artefact were acquired in all basic orientations (sagittal, transverse, and coronal, assuming head-first supine patient orientation and for both possible assignments of the readout and the phase-encode directions) for the container with and without the tested materials. The parameters of the sequences used in the experiment are described in Table 2.

To analyse the artefacts caused by metallic bracket set, it was mounted on a polymethyl methacrylate (PMMA) jaw model (SpofaDental a.s., Czech Republic), which was put in a small container $(120 \mathrm{~mm} \times 170 \mathrm{~mm} \times 90 \mathrm{~mm})$, immersed in water and fixed in a position corresponding to head-first supine patient orientation. The distortions were evaluated visually using the modified receiver operating characteristic method of distortion classification $^{25}$, since unequivocal definition of reliable quantitative measurements was not possible. This method grading scale is shown in Table 3 .

For the measurement of the artefacts caused by a single metallic bracket, the modified ASTM F211907 standard was used ${ }^{37}$. A bracket was attached by a droplet of hot melt glue (Pattex, Henkel AG \& Co. $\mathrm{KGaA}$ ) to a wooden holder and immersed in a $\mathrm{CuSO}_{4}$ solution $(1.25 \mathrm{~g} / \mathrm{l})$, used to adjust the water relaxation time $\mathrm{T} 1$ to the brain tissue values of about $1300 \mathrm{~ms}$, at the centre of a PMMA boxshaped phantom with $100 \mathrm{~mm} \times 100 \mathrm{~mm} \times 100 \mathrm{~mm}$ inner dimensions. Three PMMA cylindrical bars were positioned inside the phantom near its edges for reference. Each bracket was tested with the base oriented downwards.

Quantitative measurement of artefacts by a single bracket was evaluated in Marevisi (software for MRI and MRSI data visualization and processing by Institute for Biodiagnostics, NRC Canada; originally called WIN-MRI) ${ }^{38}$. First, the average intensity inside a selected region of interest (ROI) of the reference image (without a sample) was calculated. The ROI was selected to cover the whole extent of the artefact in the image with the sample, excluding the reference bars and the holder. Then in the bracket-containing image, all pixels with intensities differing from the ROI average of the bracket-free reference image by $30 \%$ or more were considered artefactual and were marked as black or white if they were hypo- or hyperintense, respectively.

The known bracket dimension and position relative to the reference rods were drawn in the image. The maximal physical distance between the bracket and the artefact boundary (d) in mm was evaluated and recorded as the extent of the artefact. Furthermore, the total physical area of the white and black pixels inside the ROI, excluding the bracket interior, was used to characterize the distortion area. Finally, for each image slice orientation, the maximal extent of the artefact and the maximal artefact area from all slices were evaluated.

Magnetic field interaction examination was performed in the same $1.5 \mathrm{~T}$ whole-body scanner. For the evaluation of translational attraction, the bracket was suspended on a thin nylon thread attached near its mass centre to a stand made of synthetic methacrylic resin (Dentacryl, SpofaDental a.s., Czech Republic), with a protractor mounted ${ }^{9}$. The angle of deflection from the vertical axis was determined at the position where the maximal attraction force was found ${ }^{39}$, i.e., at the edge of the magnet bore, as well as along the magnet axis. The position was identified by visual inspection. According to the ASTM F2052-06e1 standard, "if the device deflects less than $45^{\circ}$, then the magnetically induced deflection force is lower than the force on the device due to gravity (its weight). For this condition it is assumed that any risk imposed by the application of the magnetically induced force is no greater than any risk imposed by normal daily activity in the Earth's gravitational field" ${ }^{40}$.

To detect the presence of torque and displacement forces (due to eddy currents arising during table motion), the observed bracket was placed in the reference point on the millimetre paper in a Petri dish used as a holder. The bracket was tested in 6 positions with different longitudinal axis orientation relative to main static magnetic field $\mathrm{B}_{0}$ direction $\left(0-90^{\circ}\right.$ at $15^{\circ}$ increments). The holder was moved back and forth into the magnet isocenter with uniform magnetic field, then the torque and displacement were measured by comparing with reference position. 
Table 4 Comparison of artefact sizes for the orthodontic brackets tested!

\begin{tabular}{lccccc}
\hline \multirow{2}{*}{ Sequence } & $\begin{array}{c}\text { Slice } \\
\text { orient. }\end{array}$ & \multicolumn{2}{c}{$d(\mathrm{~mm})$} & \multicolumn{2}{c}{$A\left(\mathrm{~mm}^{2}\right)$} \\
\cline { 3 - 6 } & Equi. ti & Gemini & Equi. ti & Gemini \\
\hline \multirow{3}{*}{ SE } & S & 4.3 & $>50$ & 29 & 2573 \\
& C & 4.4 & $>50$ & 40 & 2620 \\
& T & 2.5 & $>50$ & 22 & 2280 \\
GE & S & 4.9 & $>50$ & 62 & $>5772$ \\
& C & 4.7 & $>50$ & 74 & $>5629$ \\
& T & 4.8 & $>50$ & 109 & 7536 \\
& $S^{*}$ & - & 48 & - & 4105 \\
\hline
\end{tabular}

$\dagger d$, the maximum distance from the bracket edge to the artefact edge; $A$, the maximum area of the artefact lessened by the area of the bracket; bracket size, Table 1; acquisition parameters, Table 2; GE:TE = $10 \mathrm{~ms}$ ("6 ms).

$\$$ S, sagittal; C, coronal; T, transversal.

For testing RF heating, we placed single brackets inside a box thermally isolated from the environment by $1 \mathrm{~cm}$ thick polystyrene and located outside the experimental small-bore $4.7 \mathrm{~T}$ scanner in a small RF coil used for mouse imaging. This coil was used to irradiate the box with a sequence of rectangular RF pulses with $\mathrm{B}_{1}=49 \mu \mathrm{T}$ at $198 \mathrm{MHz}$, which were applied for 10 min with a duty cycle of $50 \%$. The heating effect created by this RF field $\mathrm{B}_{1}$ exceeded that of turbo spin echo (TE $=13 \mathrm{~ms}$, $180^{\circ} \mathrm{AM}$ RF pulse) at $1.5 \mathrm{~T}$ by a factor $>500$ and in $3 \mathrm{~T}$ by a factor $>160$. The bracket temperature was monitored with a thermocouple with the detection sensitivity of $0.1{ }^{\circ} \mathrm{C}$.

\section{RESULTS}

For each single bracket, the maximal distance between the bracket boundary and the fringe of the artefact, as well as the maximal artefact area are listed in Table 4. In MRI imaging using the GE sequence, which is more prone to metal artefacts than the SE sequence, the linear size (d) of the distortion caused by SS bracket was by about an order of magnitude larger than that found with the Ti bracket (in sagittal images $>50 \mathrm{~mm}$ versus $4.9 \mathrm{~mm}$, in coronal images $>50 \mathrm{~mm}$ versus $4.7 \mathrm{~mm}$ and in transversal images $>50 \mathrm{~mm}$ versus $4.8 \mathrm{~mm}$ ). Furthermore, the artefacts caused by a single SS bracket often extended over the whole observed volume, i.e., over all slices and the whole ROI (Figs. 1,2), while distortions caused by the titanium bracket were just local (Figs. 3, 4). The dependence of artefact size on echo time in GE images was

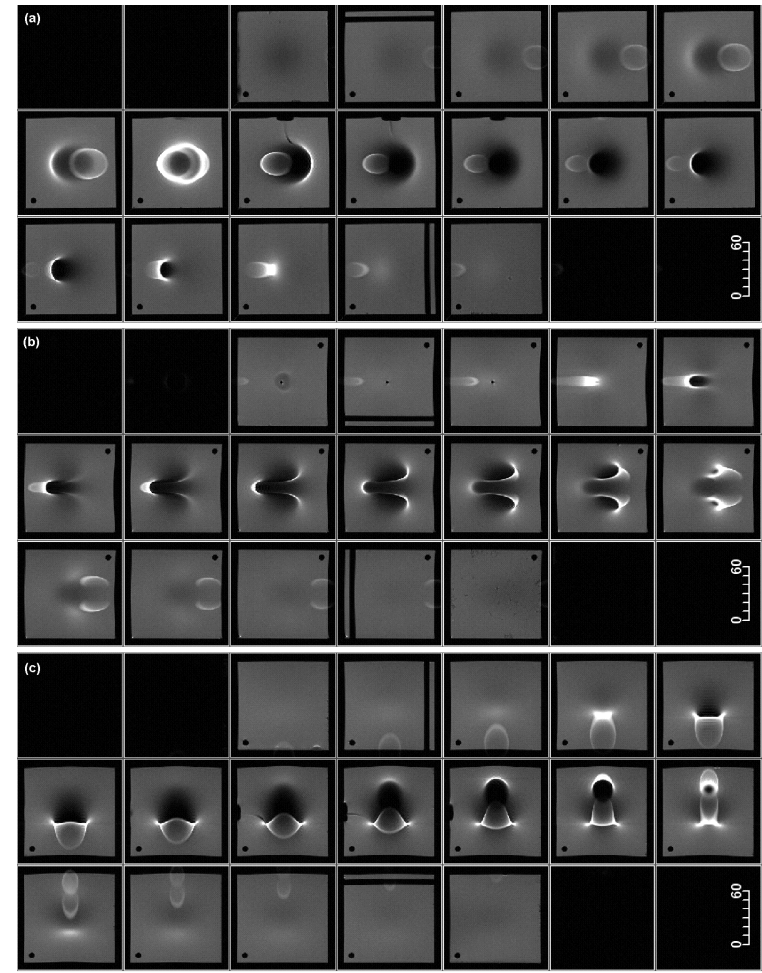

Fig. 1 SE artefacts with a single Gemini bracket the bracket size as in Table 1. Acquisition parameters: SE, 21 slices, thickness $3 \mathrm{~mm}$, gaps between slices $3 \mathrm{~mm}$, FOV $120 \mathrm{~mm} \times 120 \mathrm{~mm}$, matrix $256 \mathrm{~mm} \times 256 \mathrm{~mm}$, $\mathrm{TR}=3000 \mathrm{~ms}$, TE $=15 \mathrm{~ms}$, refocusing pulse flip angle $180^{\circ}$, bandwidth $125 \mathrm{~Hz} /$ pixel. (a) Transverse slices with LR readout. For the other gradient assignment, the artefacts were found nearly identical with axes exchanged. (b) Coronal slices with LR readout. The same artefact shapes appeared in sagittal slices with AP readout. (c) Sagittal slices with IS readout. The same artefacts were found in coronal slices with IS readout.

also observed (Fig. 5). The whole set of the SS brackets mounted on a jaw model precluded MR measurement by rendering the system incapable of adjusting the resonance frequency. Artefacts caused by titanium brackets mounted on a jaw model were considered diagnostically acceptable according to visual scale (Table 3 ), because the distortions were just local. They are displayed in Fig. 6.

It was measured that the deflection angle of the Ti bracket was $0^{\circ}$. For the SS brackets its value was more than $89^{\circ}$. No torque was detected for the Ti brackets, while for the SS brackets it could not be quantified due to Petri dish size limitations because of considerable attraction force on the magnet axis. For both brackets, the temperature increase was 


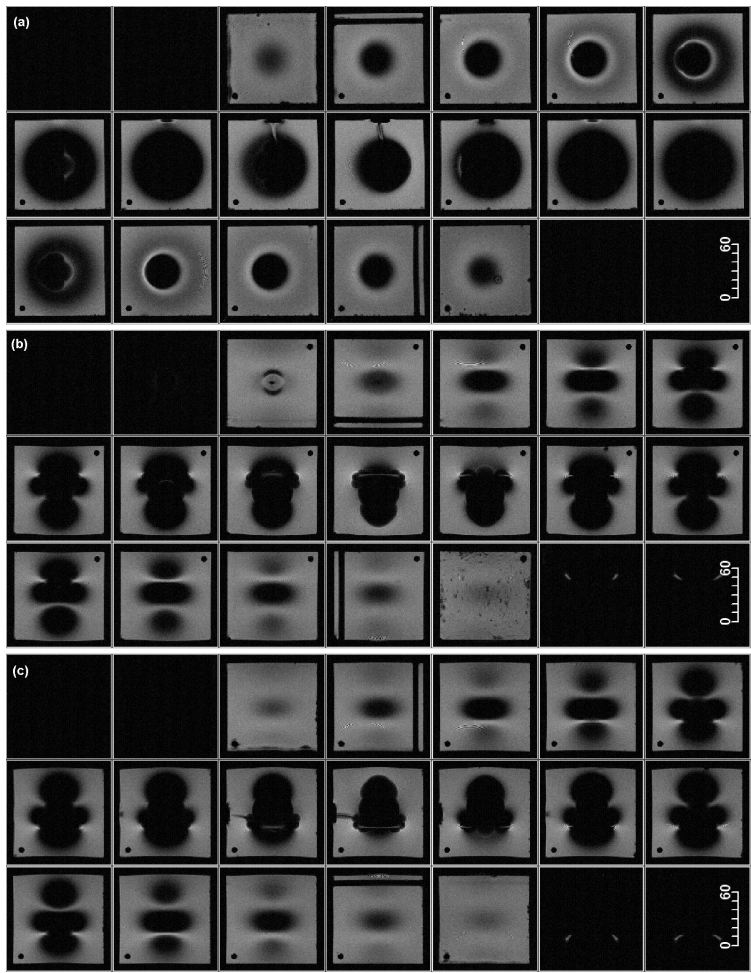

Fig. 2 GE artefacts with a single Gemini bracket the bracket size as in Table 1. Acquisition parameters: GE, 21 slices, thickness $3 \mathrm{~mm}$, gaps between slices $3 \mathrm{~mm}$, FOV $120 \mathrm{~mm} \times 120 \mathrm{~mm}$, matrix $256 \mathrm{~mm} \times 256 \mathrm{~mm}$, $\mathrm{TR}=200 \mathrm{~ms}$, TE $=10 \mathrm{~ms}$, flip angle $35^{\circ}$, bandwidth $125 \mathrm{~Hz} /$ pixel. (a) Transverse slices with LR readout. (b) Coronal slices with IS readout. (c) Sagittal slices with IS readout. No effect of gradient assignment change was observed.

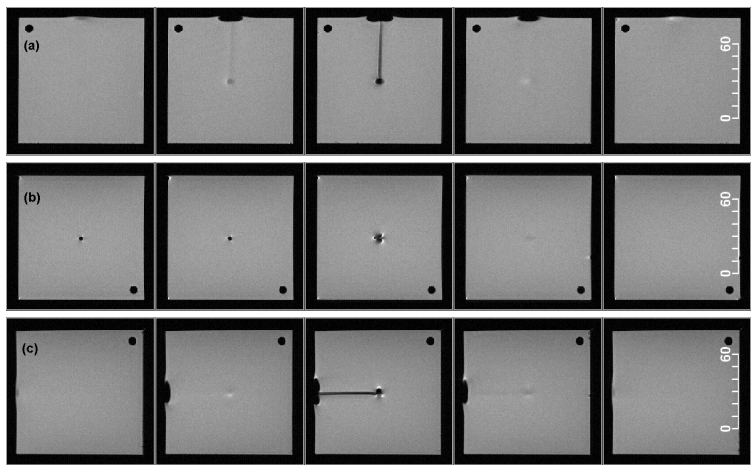

Fig. 3 SE artefacts with a single equilibrium ti bracket. The same measurement parameters as in Fig. 1. (a) Transverse slices. (b) Coronal slices. (c) Sagittal slices. Change of gradient assignment had no effect.

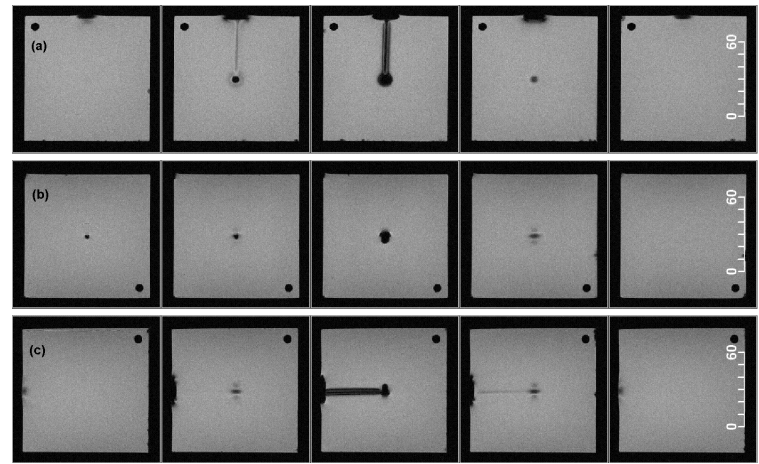

Fig. 4 GE artefacts with a single equilibrium ti bracket. The same measurement parameters as in Fig. 2. (a) Transverse slices. (b) Coronal slices. (c) Sagittal slices. Change of gradient assignment had no effect.

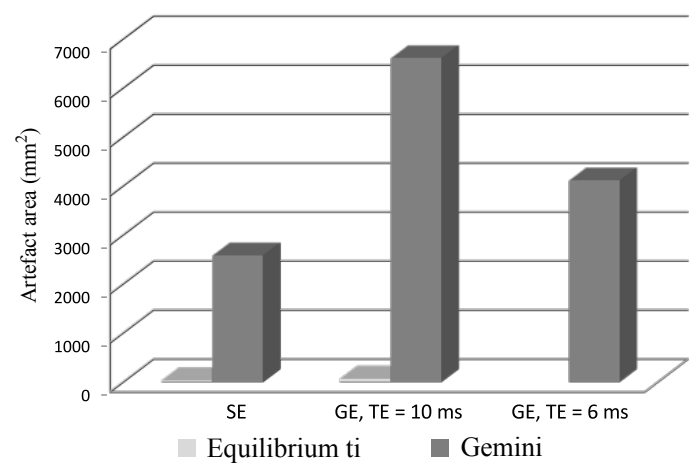

Fig. 5 Comparison of artefact areas in sagittal images for a single equilibrium ti and Gemini bracket. The column height in the graph represented the artefact area found in the sagittal images for both SE and GE images. As the artefact of GE image (TE $=10 \mathrm{~ms}$ ) for Gemini bracket extended outside of the phantom volume, the artefact area outside of the box was estimated and overall artefact area evaluated to $6600 \mathrm{~mm}^{2}$. GE images for TE $=6 \mathrm{~ms}$ were not acquired for equilibrium ti.

less than $0.1^{\circ} \mathrm{C}$.

\section{DISCUSSION}

The fact that the presence of fixed orthodontic appliances may be a substantial barrier in patient MRI due to the MRI issues, was proven by thorough scientific studies. Orthodontic arches connecting the brackets in orthodontic appliances are often ferromagnetic, so they are subjected to attraction forces and torques, which - though found still safe and bearable in the $1.5 \mathrm{~T}$ MR scanner without selfshielding - would undoubtedly be perceived un- 

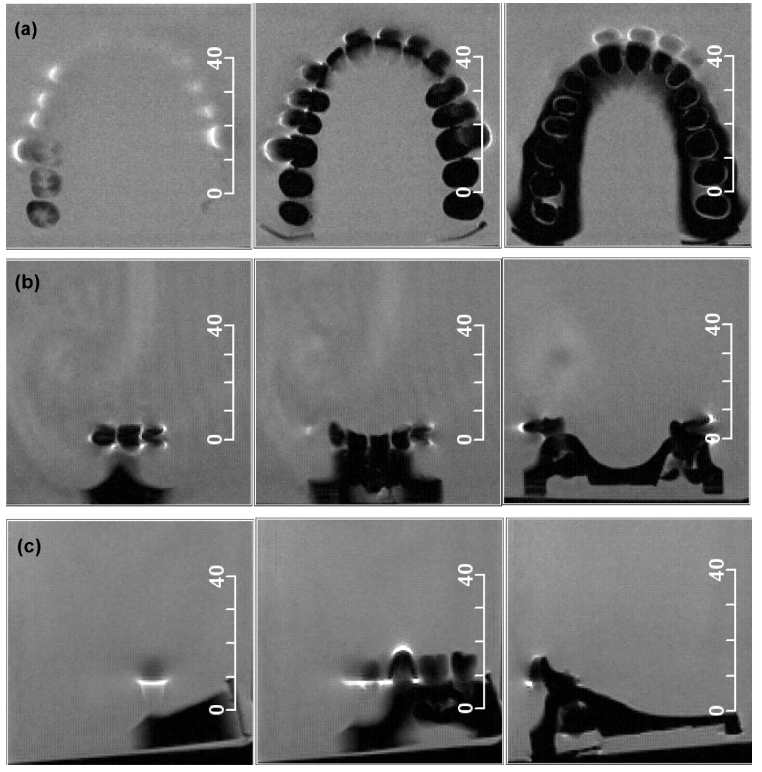

Fig. 6 SE artefacts with equilibrium ti bracket sets mounted on a jaw model. (a) Transverse slices, LR readout, IS position $42 \mathrm{~mm}, 48 \mathrm{~mm}$, and $54 \mathrm{~mm}$ from the central slice. (b) Coronal slices, LR readout, AP position $24 \mathrm{~mm}, 20 \mathrm{~mm}$, and $4 \mathrm{~mm}$ from the central slice. (c) Sagittal slices, IS readout, LR position $36 \mathrm{~mm}, 30 \mathrm{~mm}$, and $0 \mathrm{~mm}$ from the central slice. Maxillary right lateral incisor bracket was missing to allow the comparison.

comfortable by the patient and should be carefully checked for fixation. For $3 \mathrm{~T} \mathrm{MRI}^{29}$, deflection angle for NiTi and strainless steel archwires was found highly above $45^{\circ}$ (ASTM Standard F2052-06e $1^{40}$ ). Furthermore, the same arches have showed moderate and strong torque with serious alignment to the magnetic field ${ }^{29}$. More importantly, any wire loops including non-ferromagnetic ones, may pose a heating risk that is difficult to be ruled out. Fortunately, these objects can be easily removed ${ }^{26,30}$. In this regard, removable elements of orthodontic appliance were beyond the scope of the study.

Since removal of dental brackets is most undesirable because of their bonding to dental enamel, they became the subject of this in vitro study: MRI issues of metallic brackets from $\mathrm{Ti}$ and SS groups of materials were evaluated due to their dominant application in orthodontics ${ }^{33,34}$. This systematic investigation is clinically substantial for both orthodontists and radiologists.

In the present study, examination of the magnetic field interaction with Gemini brackets showed their strong attraction by the magnetic field (deflection angle $>89^{\circ}$ ), which is attributable to a ferromagnetic nature of the material used to fabricate these brackets. In the $3 \mathrm{~T}$ MRI experiment by Görgülü et al, only $13^{\circ}$ deflection angle was found for Roth bracket (Ormco) ${ }^{29}$, probably because it was less ferromagnetic. Contrarily, for equilibrium ti bracket, no translational attraction was observed. Finally, it was declared ${ }^{30,32}$ that there was no risk of detachment and displacement of orthodontic appliances if they were firmly fixed to the teeth and checked before MR examination.

In our trial, no bracket temperature increase exceeding $0.1^{\circ} \mathrm{C}$ has been found, which corresponds with other studies ${ }^{30,31}$. Contrarily, Görgülü et $\mathrm{al}^{29}$ reported a stainless steel bracket set heating up to $3.04^{\circ}$, while Hasegawa et al ${ }^{41}$ noted a temperature rise of $2.61^{\circ}$. Such heterogeneity was probably caused by different exposition time, significant variation of tested appliance design and dissimilar heat transfer conditions. Nevertheless, the above listed heating values are clinically negligible. That is why both tested materials are thought to be acceptable and MR safe according to this criterion.

According to the results of previous studies, artefacts are the major concern of MRI ${ }^{24,25,28}$. The degree of artefacts produced by metallic objects depends on many factors such as chemical composition, internal structure, physical properties, mainly magnetic susceptibility, their shape and orientation in the magnetic field. However, the orthodontist can influence only the chemical composition of the materials by choosing a specific product. Hence the results of artefact studies directly correspond with the brand name of the brackets. That is why similar studies are essential for all types of existing and perspective dental materials.

Published studies concerning MR image artefacts of orthodontic appliances contain incomparable and often controversial results. Besides metallic object parameters, distortions are also sensitive to imaging protocol and especially MRI magnetic field strength. For 0.5 T MR imaging, presence of stainless steel bracket sets did not interfere with brain ${ }^{23,26}$ and TMJ scans ${ }^{26,27}$ since the artefacts were concentrated in the condylar ${ }^{27}$ or orofacial area ${ }^{23,26}$. In $1.5 \mathrm{~T}$ MRI, stainless steel bracket sets caused significant image distortions, which rendered MRI scans non-diagnostic for teeth $^{42}$, tongue ${ }^{25,28}$, hard palate ${ }^{25}$, oral cavity ${ }^{24}$, body of the mandible ${ }^{25}$, nasal cavity ${ }^{28}$, maxillary sinus $^{24}$, all paranasal sinuses ${ }^{28}$, nasopharyn $x^{25}$, temporomandibular joints ${ }^{24,28}$, orbits/globes ${ }^{25,28}$, posterior cerebral fossa ${ }^{24}$, brain stem ${ }^{25,28}$, cerebellum $^{28}$, pituitary gland ${ }^{25}$, frontal lobe ${ }^{25,28}$, tem- 
poral lobes ${ }^{25,28}$. In this study, even without the presence of ferromagnetic arch wires, the stainless steel bracket set rendered the scanner incapable of adjusting the resonance frequency. As a result, this may cause significant difficulties, especially in diagnostics of central nervous system conditions, where MRI is the method of choice. The inability to test full-arch bracket set was probably caused by more ferromagnetic content of Gemini brackets comparing to the types tested by other investigators (Omniarch (GAC) ${ }^{24}$, MicroArch (GAC) ${ }^{25}$, unknown brand name ${ }^{28}$, Dentaurum ${ }^{42}$, Victory Series (3M Unitek) ${ }^{43}$ ). It is strongly recommended to remove all stainless steel brackets prior to MRI examination $^{24,44}$ and, evidently, this conclusion is applicable to the Gemini brackets. However, there is an alternative approach such as usage of other diagnostic method (CT, ultrasound, X-ray and other), when applicable. Additionally, the presence of less ferromagnetic orthodontic brackets would require specific metallic artefact reduction techniques ${ }^{45,46}$.

In 1.5 T MRI, single stainless steel bracket originated artefacts of incommensurable dimensions: according to Wylezinska et al, 3M Unitec bracket has showed $3 \mathrm{~mm}$ distortions ${ }^{47}$, while Zachriat et al have reported artefacts in range from $44-74 \mathrm{~mm}$ for $3 \mathrm{M}$ Unitec brackets ${ }^{28}$. In this study, single Gemini bracket caused artefacts exceeding $50 \mathrm{~mm}$, their exact sizes were not determined since they extended outside of the phantom volume. These findings support the results of Zachriat et $\mathrm{al}^{28}$ and are primarily valuable for comparative investigations of different bracket systems. They can also be used to predict the presence of artefacts in vivo.

Corrosion of stainless steel brackets and nickel leaching, which is a great concern for patient health due to allergic reactions and cytotoxic effects, stimulated the manufacturers to use other materials, so titanium brackets were developed ${ }^{7}$. Up to date, these brackets were not studied so extensively as stainless steel ones. In 1.5 T MRI, TitaniumOrthos bracket set (Ormodent) caused small artefacts in the close proximity to the appliance ${ }^{24}$. Our results of equilibrium ti bracket sets are in agreement with the results of Beau et al. Besides, according to the results of the present study, single equilibrium ti bracket produced image distortions in range from 2.5-4.9 mm. In our opinion, these weakly paramagnetic brackets may be considered as acceptable even for MRI of the maxillofacial region.

Several limitations of this study should be considered. Artefacts were investigated in $1.5 \mathrm{~T}$ magnetic field, which is the most clinically available ${ }^{28}$.
However, since no specific software is available for evaluation of artefact quality and $\operatorname{size}^{28}$ and the quality of artefact measurement methods differs significantly, the comparison of the results with other $1.5 \mathrm{~T}$ experiments is quite complicated. Obviousely, they cannot be transferred to higher field strength measurements. Only a few studies of artefacts due to orthodontic brackets in 3 T MRI are available in the scientific literature. In the clinical 3.0 T MRI study by Cassetta et $\mathrm{al}^{43}$., stainless steel brackets severely affected the diagnostic quality of cervical region, cervical vertebrae, paranasal sinuses, head and neck MRI scans. MRI images of the brain and temporomandibular joint regions were found diagnostic. Zhylich et $\mathrm{al}^{48}$ reported that stainless steel brackets and buccal tubes rendered images non-diagnostic in head MR images for sagittal T1-weighted, axial gradient-recalled, and axial diffusion-weighted sequences. Hence further studies would be necessary to evaluate orthodontic brackets in 3 T MRI. Consequently, artefact size vary depending on MRI technique. Optimization of imaging parameters, such as short echo time in spin echo sequence, and short echo spacing in turbo spin echo sequence allow mitigation of signal loss ${ }^{47}$. Furthermore, several new techniques were designed to reduce metal artefacts (MARS ${ }^{45}$, SEMAC ${ }^{46}$ ). In this investigation, standard settings, which are used in daily practice, were selected to demonstrate the worst case of artefact presence. Furthermore, the measurements were far from covering all existing brackets types. Only metallic brackets were tested since it was reported that ceramic and plastic orthodontic brackets did not produce image artefacts. Just one brand of brackets from each material group (stainless steel and titanium), which represented the most commonly used fixed orthodontic appliances, was investigated. Despite these limitations, the present study resulted in clinically important findings on assessment of MR image quality degradation due to the presence of orthodontic brackets.

In conclusion, the artefact size is the key criterion of MRI issues of orthodontic brackets. Taking into consideration the artefact size, out of the brackets tested only equilibrium ti brackets may be recommended in clinical practice as acceptable for 1.5 T MRI. Stainless steel brackets were stated as incompatible with MRI of the same magnetic field strength. Unfortunately, artefacts are no longer considered in the contemporary standard as an issue. However, even if clinically important anatomical areas are located far from the oral cavity, MR image distortions due to the mentioned objects may lead 
to misdiagnosis. Undoubtedly, artefacts should be a subject of closer attention and detailed study. First judgment of MRI issues of the material may be done by simply testing its attractiveness by the magnetic field.

Acknowledgements: The authors thank to Petr Krupa, MD (St Anne's University Hospital, Brno, Czech Republic) for providing the MR system used in this project and to Prof. ing. Karel Bartušek, DrSc for measurement of brackets heating. Preparation of the article was supported by MEYS CR (LO1212) together with EC (ALISI No. CZ.1.05/2.1.00/01.0017), by ASCR (RVO:68081731) and by Charles University in Prague (PROGRES Q29/LF1).

\section{REFERENCES}

1. Stark DD, Bradley WG Jr (1999) Magnetic Resonance Imaging, 3rd edn, Mosby, St Louis.

2. White SC, Pharoah MJ (2000) Oral Radiology: Principles And Interpretation, 4th edn, Mosby, St Louis.

3. Walker REA, Eustace SJ (2001) Whole-body magnetic resonance imaging: techniques, clinical indications, and future applications. Semin Musculoskelet Radiol 5, 5-20.

4. Gray CF, Redpath TW, Smith FW, Staff RT (2003) Advanced imaging: Magnetic resonance imaging in implant dentistry. Clin Oral Implants Res 14, 18-27.

5. Eustace SJ, Nelson E (2004) Whole body magnetic resonance imaging. BMJ 328, 1387-8.

6. Shellock FG, Crues JV (2004) MR procedures: biologic effects, safety, and patient care. Radiology 232, 635-52.

7. Iijima M, Zinelis S, Papageorgiou SN, Brantley W, Eliades T (2016) Orthodontic brackets. In: Eliades T, Brantley WA (eds), Orthodontic Applications of Biomaterials: A Clinical Guide, Woodhead Publishing, pp 75-96.

8. Shellock FG, Spinazzi A (2008) MRI safety update 2008: part 2, screening patients for MRI. AJR Am J Roentgenol 191, 1140-9.

9. New PFJ, Rosen BR, Brady TJ, Buonanno FS, Kistler JP, Burt CT, Hinshaw WS, Newhouse JH, et al (1983) Potential hazards and artefacts of ferromagnetic and nonferromagnetic surgical and dental materials and devices in nuclear magnetic resonance imaging. $\mathrm{Ra}$ diology 147, 139-48.

10. Schenck JF (1996) The role of magnetic susceptibility in magnetic resonance imaging: MRI magnetic compatibility of the first and second kinds. Med Phys 23, 815-50.

11. Shafiei F, Honda E, Takahashi H, Sasaki T (2003) Artifacts from dental casting alloys in magnetic resonance paging. $J$ Dent Res 82, 602-6.

12. Abbaszadeh K, Heffez LB, Mafee MF (2000) Effect of interference of metallic objects on interpretation of T1-weighted magnetic resonance images in the maxillofacial region. Oral Surg Oral Med Oral Pathol Oral Radiol Endod 89, 759-65.

13. Starčuková J, Starčuk Z, Hubálková H, Linetskiy I (2008) Magnetic susceptibility and electrical conductivity of metallic dental materials and their impact on MR imaging artifacts. Dent Mater 24, 715-23.

14. Vikhoff B, Ribbelin S, Köhler B, Ekholm S, Borrman H (1995) Artefacts caused by dental filling materials in MR imaging. Acta Radiol 36, 323-5.

15. ASTM International (2008) Standard practice for marking medical devices and other items for safety in the magnetic resonance environment ASTM F250308, ASTM International, West Conshohocken, PA.

16. Shellock FG (2002) Magnetic resonance safety update 2002: implants and devices. J Magn Reson Imaging 16, 485-96.

17. Shellock FG (2001) Metallic neurosurgical implants: evaluation of magnetic field interactions, heating, and artifacts at 1.5 Tesla. J Magn Reson Imaging 14, 295-9.

18. Davis PL, Crooks L, Arakawa M, McRee R, Kaufman L, Margulis AR (1981) Potential hazards in NMR imaging: heating effects of changing magnetic fields and RF fields on small metallic implants. AJR Am J Roentgenol 137, 857-60.

19. US-FDA (1997) A primer on medical device interactions with magnetic resonance imaging systems, Food and Drug Administration, US Department of Health and Human Service. Available online: www.cognitiveneuro.org/SafetyReadings/ FDAExternalDeviceGuidance\%201997.pdf.

20. Hubálková H, La Serna P, Linetskiy I, Dostálová T (2006) Dental alloys and magnetic resonance imaging. Int Dent $J$ 56, 135-41.

21. Lissac M, Coudert JL, Briguet A, Amiel M (1992) Disturbances caused by dental materials in magnetic resonance imaging. Int Dent $J$ 42, 229-33.

22. Eggers G, Rieker M, Kress B, Fiebach J, Dickhaus H, Hassfeld S (2005) Artefacts in magnetic resonance imaging caused by dental material. Magma Magn Reson Mater Phys Biol Med 18, 103-11.

23. Hinshaw DB, Holshouser BA, Engstrom HI, Tjan AHL, Christiansen EL, Catelli WF (1988) Dental material artefacts on MR images. Radiology 166, 777-9.

24. Beau A, Bossard D, Gebeile-Chauty S (2015) Magnetic resonance imaging artefacts and fixed orthodontic attachments. Eur J Orthod 37, 105-10.

25. Elison JM, Leggitt VL, Thomson M, Oyoyo M, Wycliffe ND (2008) Influence of common orthodontic appliances on the diagnostic quality of cranial magnetic resonance images. Am J Orthod Dentofac Orthop 134, 563-72.

26. Sadowsky PL, Bernreuter W, Lakshminarayanan AV, Kenney P (1988) Orthodontic appliances and magnetic resonance imaging of the brain and temporomandibular joint. Angle Orthod 58, 9-20. 
27. Okano Y, Yamashiro M, Kaneda T, Kasai K (2003) Magnetic resonance imaging diagnosis of the temporomandibular joint in patients with orthodontic appliances. Oral Surg Oral Med Oral Pathol Oral Radiol Endod 95, 255-63.

28. Zachriat C, Asbach P, Blankenstein KI, Peroz I, Blankenstein FH (2015) MRI with intraoral orthodontic appliance-a comparative in vitro and in vivo study of image artefacts at $1.5 \mathrm{~T}$. Dentomaxillofac Radiol 44, 20140416.

29. Görgülü S, Ayyıldız S, Kamburoğlu K, Gökce S, Ozen $\mathrm{T}$ (2014) Effect of orthodontic brackets and different wires on radiofrequency heating and magnetic field interactions during 3-T MRI. Dentomaxillofac Radiol 43, 20130356.

30. Yassi K, Ziane F, Bardinet E, Moinard M, Veyret B, Chateil JF (2007) Évaluation des risques d'échauffement et de déplacement des appareils orthodontiques en imagerie par résonance magnétique. $J$ Radiol 88, 263-8.

31. Regier M, Kemper J, Kaul MG, Feddersen M, Adam G, Kahl-Nieke B, Klocke A (2009) Radiofrequencyinduced heating near fixed orthodontic appliances in high field MRI systems at 3.0 Tesla. J Orofac Orthop 70, 485-94.

32. Kemper J, Priest AN, Schulze D, Kahl-Nieke B, Adam G, Klocke A (2007) Orthodontic springs and auxiliary appliances: assessment of magnetic field interactions associated with $1.5 \mathrm{~T}$ and $3 \mathrm{~T}$ magnetic resonance systems. Eur Radiol 17, 533-40.

33. Keim RG, Gottlieb EL, Nelson AH, Vogels DS (2008) JCO study of orthodontic diagnosis and treatment procedures - part 1: results and trends. J Clin Orthod 42, 625-40.

34. Banks P, Elton V, Jones Y, Rice P, Derwent S, Odondi L (2010) The use of fixed appliances in the UK: a survey of specialist orthodontists. J Orthod 37, 42-55.

35. Young IR, Bydder GM (2003) Magnetic resonance: new approaches to imaging of the musculoskeletal system. Physiol Meas 24, R1-23.

36. Sasaki $\mathrm{M}$, Inoue $\mathrm{T}$, Tohyama $\mathrm{K}$, Oikawa $\mathrm{H}$, Ehara S, Ogawa A (2003) High-field MRI of the central nervous system: current approaches to clinical and microscopic imaging. Magn Reson Med Sci 2, 133-9.

37. ASTM International (2013) Standard test method for evaluation of MR image artifacts from passive implants ASTM F2119-07, ASTM International, West Conshohocken, PA.
38. Starcukova J, Kozlowski P, Starcuk Z, Saunders J K, Germanus A, Thiele H (1997) WIN-MRI: PC software for imaging and spectroscopic imaging data processing and presentation. In: 1st Kraków - Winnipeg Workshop on Biomedical Applications of MRI and MRS, Komitet Badan Naukovych, Kraków.

39. Kagetsu NJ, Litt AW (1991) Important considerations in measurement of attractive force on metallic implants in MR imagers. Radiology 179, 505-8.

40. ASTM International (2006) Standard test method for measurement of magnetically induced displacement force on medical devices in the magnetic resonance environment ASTM F2052-06e1, ASTM International, West Conshohocken, PA.

41. Hasegawa M, Miyata K, Abe Y, Ishigami T (2013) Radiofrequency heating of metallic dental devices during 3 T MRI. Dentomaxillofac Radiol 42, 20120234.

42. Tymofiyeva O, Vaegler S, Rottner K, Boldt J, Hopfgartner AJ, Proff PC, Richter EJ, Jakob PM (2013) Influence of dental materials on dental MRI. Dentomaxillofac Radiol 42, 20120271.

43. Cassetta M, Pranno N, Stasolla A, Orsogna N, Fierro D, Cavallini C, Cantisani V (2017) The effects of a common stainless steel orthodontic bracket on the diagnostic quality of cranial and cervical 3T-MR images: a prospective, case-control study. Dentomaxillofac Radiol 46, 20170051.

44. Poorsattar-Bejeh Mir A, Rahmati-Kamel M (2016) Should the orthodontic brackets always be removed prior to magnetic resonance imaging (MRI)? J Oral Biol Craniofac Res 6, 142-52.

45. Olsen RV, Munk PL, Lee MJ, Janzen DL, MacKay AL, Xiang QS, Masri B (2000) Metal artifact reduction sequence: early clinical applications. Radiographics 20, 699-712.

46. Lu W, Pauly KB, Gold GE, Pauly JM, Hargreaves BA (2009) SEMAC: slice encoding for metal artifact correction in MRI. Magn Reson Med 62, 66-76.

47. Wylezinska M, Pinkstone M, Hay N, Scott AD, Birch MJ, Miquel ME (2015) Impact of orthodontic appliances on the quality of craniofacial anatomical magnetic resonance imaging and real-time speech imaging. Eur J Orthod 37, 610-7.

48. Zhylich D, Krishnan P, Muthusami P, Rayner T, Shroff M, Doria A, Tompson B, Lou W, et al (2017) Effects of orthodontic appliances on the diagnostic quality of magnetic resonance images of the head. Am J Orthod Dentofacial Orthop 151, 484-99. 